Check for updates

Cite this: RSC Adv., 2017, 7, 17889

\title{
Sub-micron silk fibroin film with high humidity sensibility through color changing
}

\author{
Qingsong Li, ${ }^{a}$ Ning Qi, ${ }^{a}$ Yu Peng, ${ }^{a}$ Yafeng Zhang, ${ }^{e}$ Lei Shi, ${ }^{b}$ Xiaohua Zhang, ${ }^{\text {*c }}$ \\ Yuekun Lai, ${ }^{a}$ Kai Wei, ${ }^{a}$ Ick Soo Kim ${ }^{d}$ and Ke-Qin Zhang ${ }^{* a}$
}

Use of structural colors for humidity sensors has great potential owing to their not being power driven and having distinct stimulus/color variation properties, but unfortunately most 1D, 2D or 3D photonic crystals have subtle nanostructures which are difficult to fabricate. Here we report a one-layer sub-micron thin film with bright color and high sensitivity to humidity, by spin coating of silk fibroin (SF) solution. The optical properties of the SF film caused by thin film interference can be easily tuned by the coating rates. Due to the high hydrophilicity of SF, the film exhibits fast responses with evident color variation in $5 \mathrm{~s}$. And combined with the large peak red-shifts for more than $130 \mathrm{~nm}$, such a thin film is superior to many other multilayered or photonic crystal based humidity sensors. Considering the good reversibility and durability, these low-cost but highly efficient SF spin coating sensors can realize colorimetric detection of humidity like $\mathrm{pH}$ indicator papers, and may have great potential in applications for anti-counterfeit labeling.

Received 20th December 2016

Accepted 14th March 2017

DOI: 10.1039/c6ra28460d

rsc.li/rsc-advances

swelling of their long, flat, multilayered scales after water

\section{Introduction}

The phenomenon of structural color was first observed on peacock tail feathers, by Robert Hooke in the 17 th century. ${ }^{1}$ Since then, structural color has attracted considerable attention due to its advantageous dye-free coloration and resilience to fading. Structural color widely exists in nature as a result of light interaction with various periodically patterned nanostructures, including diffraction grating, photonic crystals, and multilayer interference. $^{2-8}$ For example, the spectacular structural coloration of the male Sapphirinid copepods is generated via the regularly alternating layers of hexagonally-shaped guanine crystals and cytoplasm found on their bodies. ${ }^{9}$ The color varies when viewed from different angles and extends to the ultraviolet region, making their bodies virtually invisible during spiral swimming. The elytra of longhorn beetles Tmesisternus isabellae can change color from golden in a dry environment to red when wet. This is due to the optical interference caused by the

${ }^{a}$ National Engineering Laboratory for Modern Silk, College of Textile and Clothing Engineering, Soochow University, Suzhou 215123, China. E-mail: kqzhang@suda. edu.cn

${ }^{b}$ Department of Physics, Key Laboratory of Micro and Nano Photonic Structures (MOE), Key Laboratory of Surface Physics, Fudan University, Shanghai 200433, China

'Suzhou Institute of Nano-Tech and Nano-Bionics, Chinese Academy of Sciences, Ruoshui Road 398, Suzhou 215123, China. E-mail: xhzhang2009@sinano.ac.cn

${ }^{d}$ Nano Fusion Technology Research Lab, Interdisciplinary Cluster for Cutting Edge Research (ICCER), Division of Frontier Fibers, Institute for Fiber Engineering (IFES), Shinshu University, Ueda, Nagano 386 8567, Japan

${ }^{e}$ National Laboratory for Infrared Physics, Shanghai Institute of Technical Physics, Chinese Academy of Sciences, Shanghai, 200083, China infiltration. ${ }^{10}$ The most well-known display of the structural color effect is the active color change of chameleons, which is produced by tuning a lattice of guanine nanocrystals in their skin in response to the external environment. ${ }^{11}$ The unique structural colors of these aforementioned organisms provide them with visually striking appearances, allowing them to protect themselves from predators or making them better suited to environmental changes.

Optical multilayer structures are notable as a simple, yet important technique for achieving structural coloration. This structure consists of multiple layers of periodically stacked materials with varying refractive indices. Periodic variationinduced interference can even produce results for one-layer structures consisting of simple thin films such as soap bubbles and oil film on water; varying characteristics such as thickness can produce rainbow-like iridescence. ${ }^{12}$ Compared to photonic crystals, one-layer or multilayer thin films can easily be fabricated via various comparatively simpler methods. ${ }^{13}$ As the optical properties of these films highly depend on refractive index and layer thickness, they can be customized for specific device applications via variation of film thickness and introduction of other materials. Functional devices can be fabricated using bottom-up approaches based on dip- or spin-coating techniques. ${ }^{\mathbf{1 4 , 1 5}}$ Such tunable coloration opens up applications in controllable release, ${ }^{16}$ displays, ${ }^{17-19}$ stimuli responsive sensors, ${ }^{20,21}$ optical coatings for lenses ${ }^{22-24}$ and so on. Generally, multilayer thin films are composed of inorganic materials with high refractive indices such as $\mathrm{TiO}_{2}$ or $\mathrm{SiO}_{2}$. However, while these materials usually possess good optical properties, they are 
difficult to tune, as they show physicochemistry stability, and have limited variation in film thickness or period in response to external stimuli. ${ }^{25}$ Incorporation of organic, structurally flexible materials can greatly improve the responsivity, but the fabrication of these hybrid films still requires laborious stacking steps. Therefore, it is necessary to develop facile organic polymers with simpler preparation, such that straightforward fabrication of thin films with tunable colors and highly sensitive response to external stimuli can be achieved.

As a natural biomaterial, silk fibroin is environment-friendly and totally biodegradable. Owing to its favourable material properties, SF has been used for many high performance optical devices. ${ }^{26-29}$ Its easy self-crosslinking and controllable stability make it programmable with desired properties under certain conditions. ${ }^{30}$ In this paper, we report a novel SF-based submicron thin film that exhibits superior humidity-responsive coloration. The film can be rapidly prepared by spin-coating the SF solution, and film interference can be controlled via spin-coating rate. In wet environments, the SF film can quickly absorb water and expand. As a result, there is a remarkable red shift of the reflection spectrum: greater than $130 \mathrm{~nm}$ upon achieving over $90 \%$ humidity. The color change simultaneously increases with humidity in only several seconds, and the film blue shifts back to its original hue immediately after being placed in a dry environment. Compared with many other silk optical sensors which are complicated either with multicomponent or hierarchical structures, ${ }^{31-33}$ such simple and low-cost humidity sensors not only show good reversibility and durability, but also can realize fast colorimetric detection of humidity, similarly to $\mathrm{pH}$ indicator paper, as well as applications in anti-counterfeit labelling.

\section{Experimental details}

\subsection{Preparation of silk fibroin solution}

Cocoons of Bombyx mori silkworm were cut into $10 \mathrm{~mm} \times 10$ $\mathrm{mm}$ pieces and boiled in an aqueous solution of $0.5 \%(\mathrm{w} / \mathrm{v})$ $\mathrm{Na}_{2} \mathrm{CO}_{3}$ for $40 \mathrm{~min}$, then rinsed thoroughly with distilled water to remove the glue-like sericin proteins. The extracted fibroin bundles were dried in an oven overnight and then dissolved in $9.3 \mathrm{~mol} \mathrm{~L}^{-1} \mathrm{LiBr}$ solution at $60^{\circ} \mathrm{C}$ for $1 \mathrm{~h}$. The obtained solution was dialyzed in deionized water using a cellulose dialysis membrane (MWCO 6000-8000 Da, Spectra/Por, USA) at room temperature for 3 days to remove LiBr. The dialyzed silk solution was then centrifuged at $10000 \mathrm{rpm}$ for $20 \mathrm{~min}$. Finally, the supernatant with a concentration of $7 \mathrm{wt} \%$ was collected and stored at $4{ }^{\circ} \mathrm{C}$ for later usage.

\subsection{Preparation of thin films}

The SF films were prepared by spin coating the $7 \mathrm{wt} \%$ aqueous solution at 1500, 2000, 2500, 3000, and $3500 \mathrm{rpm}$ for $40 \mathrm{~s}$, onto $20 \mathrm{~mm} \times 20 \mathrm{~mm}$ silicon wafers, using a WS-650-23NPP Spin Coater (Laurell Technologies Corp. USA). The film thickness was adjusted based on the coating rate, as discussed in the main text. After spin-coating, all films were solidified in air at room temperature without any heating treatment.

\subsection{Characterization}

A Canon EOS 700D digital camera was used to photograph the films. FTIR spectra were recorded in the range $2000-4000 \mathrm{~cm}^{-1}$ with a Thermo Nicolet 5700 FTIR spectrometer (Thermo Fisher Scientific Inc., USA) to analyse the fibroin conformation. The crystalline structure of the samples was examined using X-ray diffraction (XRD, PANalytical X'pert-PRO, Netherland) in a $2 \theta$ range from $4^{\circ}$ to $60^{\circ}$. A PG2000-Pro spectrometer (Idea Optics Co., Ltd., China) equipped with a UV-VIS-NIR light source was used to detect the reflectance spectrum within $200-1100 \mathrm{~nm}$ at normal incidence. The film thickness and refractive index was measured using an alpha-SE spectroscopic ellipsometer (J. A. Woollam Co., Inc., USA) under various humidities between $\sim 25 \%$ and $\sim 80 \%$ by blowing wet air (by pumping wet air, water molecules taken from water easily condensate on the film surface when humidity is higher than $\sim 80 \%$ ). To obtain surface information, atomic force microscopy (AFM) characterization was applied in tapping mode with a Dimension Icon AFM system (Bruker Nano Inc., USA). Scanning electron microscopy (SEM) images were obtained by an S-4800 field emission microscope (Hitachi Ltd., Japan). The contact angle of water droplets on the SF film was measured by a DSA100 drop shape analysis system (Krüss GmbH, Germany). For the anticounterfeiting label, the Au film approximately $2-3 \mathrm{~nm}$ in thickness was sputtered using an E-1045 ion sputter and carbon coating unit for $15 \mathrm{~s}$ with a rate of $10 \mathrm{~nm} \mathrm{~min} \mathrm{~m}^{-1}$ (Hitachi Ltd., Japan).

For the reflection measurement, different levels of humidity inside the small chamber was obtained using different saturated salt solutions at about $21{ }^{\circ} \mathrm{C}$, i.e., $\mathrm{LiBr}, \mathrm{MgCl}_{2}, \mathrm{NH}_{4} \mathrm{NO}_{3}$, $\mathrm{KCl}$, or $\mathrm{Na}_{2} \mathrm{CO}_{3}$. Relative humidity levels of $7 \%, 33 \%, 67 \%, 85 \%$, and $92 \%$ were studied. The SF films were placed inside the chamber, at 3-5 $\mathrm{mm}$ above the liquid surface. For the reproducibility test, the humidity inside the $20 \mathrm{~cm} \times 20 \mathrm{~cm} \times 20 \mathrm{~cm}$ glass box was controlled by pumping dry nitrogen gas or wet air, set to vary between $\sim 10 \%$ and $\sim 90 \%$. The humidity was calibrated by a DSR-TH hygrometer (Zoglab Microsystem Co., Ltd, China). All the experiments were carried out at normal atmosphere.

To obtain the water-uptake property, the SF film was prepared at first by evaporating $7 \mathrm{wt} \%$ SF solution in fume hood at about $25{ }^{\circ} \mathrm{C}$ for more than 3 days. The relative humidities $(\mathrm{RH})$ of $7,33,67,85,92 \%$ was provided by storing saturated solutions as mentioned above in a closed chamber. All the samples were encapsulated in the chamber above the solution for more than $12 \mathrm{~h}$ to make sure they absorb water totally. After that the samples were taken out to weigh the mass within $10 \mathrm{~s}$. In order to get the dry weight of all the samples, they were dried in vacuum oven at $140{ }^{\circ} \mathrm{C}$ for more than $90 \mathrm{~min}$ to remove the water.

\section{Results and discussion}

The SF solution was obtained from cocoons of the Bombyx mori silkworm, which was processed through degumming, dissolution and dialysis. By spin-coating the $7 \mathrm{wt} \%$ aqueous SF solution 
at various rates of $1500,2000,2500,3000$, and $3500 \mathrm{rpm}$, submicron one-layer films were obtained with thicknesses of $\sim 414,312,258,207$, and $187 \mathrm{~nm}$ respectively. These films exhibited bright colors from violet to light orange (Fig. 1a, inset) under ambient conditions, and the reflection spectra collected on the centre of the thin films displayed multiple peaks, owing to the constructive interference over the wide spectrum of incident light ranging from 200 to $1100 \mathrm{~nm}$ (Fig. 1a). The Si wafer is smooth and high light-reflecting, it is favourable for SF film to display vivid interference color. In principle, the film color can be adjusted across entire visible spectrum just by carefully tuning the coating rate. However, the films suffered from non-uniformity at the film edge, or for coating rates below $2000 \mathrm{rpm}$. In the following discussion, most measurements were performed on the samples obtained at $3000 \mathrm{rpm}$, corresponding with a film color of bright yellow, which allowed for obvious visible detection of red and blue shifting.

The hydrophilic property of SF can affect the films' ability to absorb water. As shown in Fig. 1b, the contact angle of a water droplet on the SF film is only $\sim 65^{\circ}$, and it decreased quickly to $\sim 27^{\circ}$ within $50-70 \mathrm{~s}$, corresponding to good hydrophilicity and high water absorbability. This indicates that the SF films have rapid response to changes in humidity. Furthermore, the SF films have an extremely flat surface, and thus, uniform film thickness that is favourable for displaying bright colors. Fig. 1c shows images from SEM and surface mapping by AFM. Within a length scale of $20 \mu \mathrm{m}$, the surface corrugation was measured to be within $\sim 2.8 \mathrm{~nm}$ and the root mean square roughness $\left(R_{\mathrm{q}}\right)$ was just $0.79 \mathrm{~nm}$; the $\mathrm{SF}$ film is much flatter than some other interference films (for example, roughness was approximately $\sim 3.7 \mathrm{~nm}$ in ref. 34 and $\sim 20 \mathrm{~nm}$ in ref. 35 ). The flatness is a result of the SF molecules forming a tightly crosslinked network with a nanometer scale pore size.

The SF films' sensitivity to humidity changes was characterized in a small closed chamber constructed using a Petri dish (90 $\mathrm{mm}$ in diameter and $15 \mathrm{~mm}$ in height). A $50 \mathrm{~mL}$ saturated salt ( $\mathrm{LiBr}, \mathrm{MgCl}_{2}, \mathrm{NH}_{4} \mathrm{NO}_{3}, \mathrm{KCl}$, or $\mathrm{Na}_{2} \mathrm{CO}_{3}$ ) solution was placed inside the chamber, and the SF film was placed approximately 3-5 $\mathrm{mm}$ above the liquid surface. As the chamber was isolated from outer atmosphere, it was possible to control the humidity according to the fixed partial vapor pressure for a certain salt solution at constant temperature. The humidity was maintained between $7 \%$ and $92 \%$. Due to the high hydrophilicity, it was possible to quickly characterize the film's sensitivity to humidity based on the shifting of the reflection peak when the decrease of peak intensity was small; larger shifts in peak wavelength in the visible light corresponded with clearer coloration changes and better identification of humidity. Fig. 2a shows a series of reflection spectra for SF films (coating rate $3000 \mathrm{rpm}$ ) kept under humidity of $7 \%, 33 \%, 67 \%, 85 \%$, and
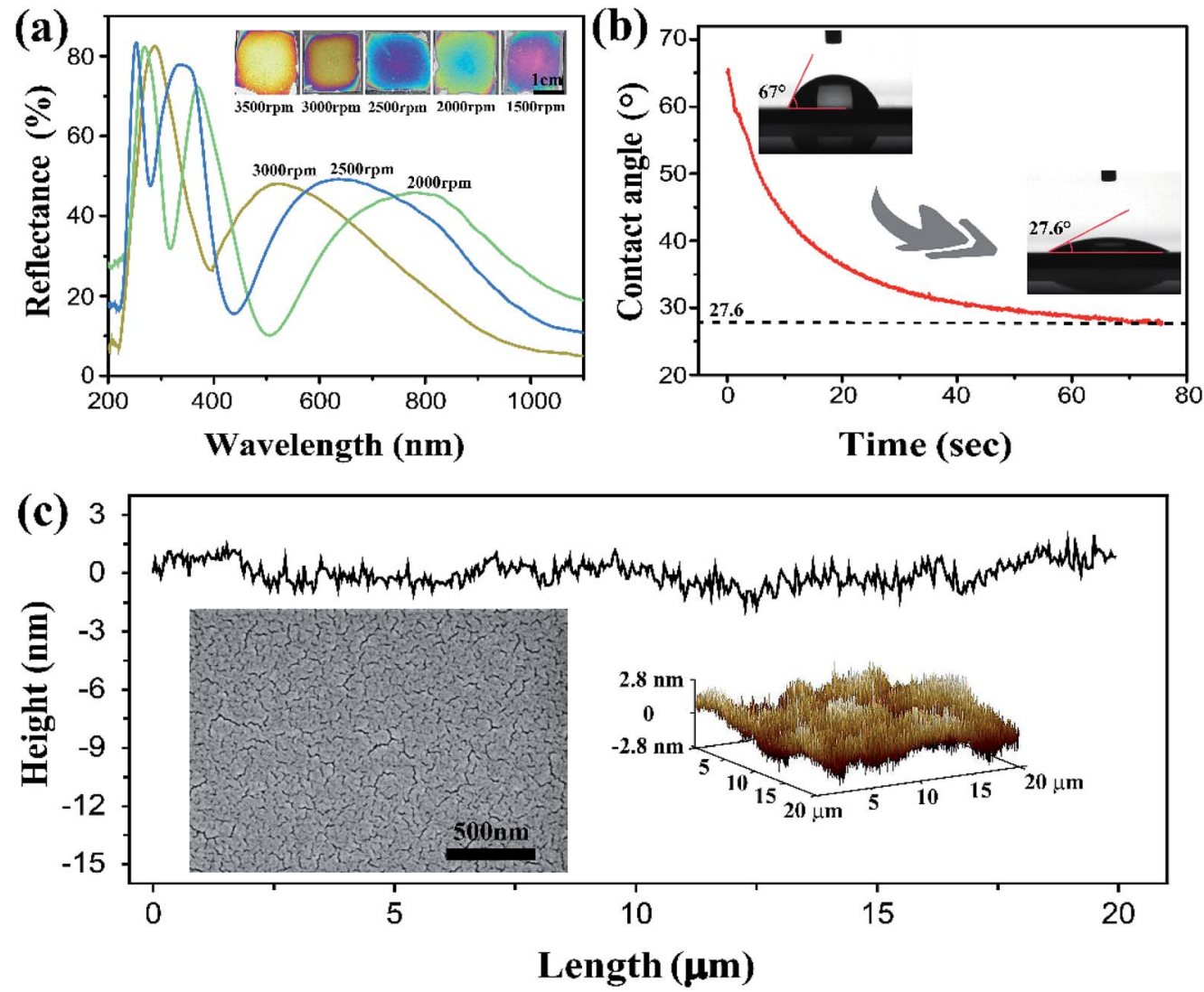

Fig. 1 Characterization of as-produced SF films. (a) Reflection spectra for the films obtained at different spin-coating rates and their optical images (insets). (b) Time evolution of water contact angle on a SF film. (c) AFM height profile of the film obtained at $3000 \mathrm{rpm}$. The corresponding SEM image and surface mapping are shown as insets. 
(a)

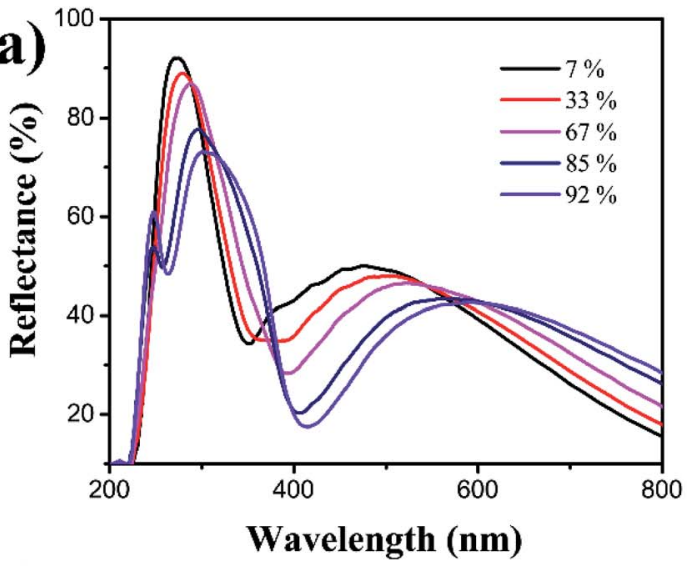

(c)

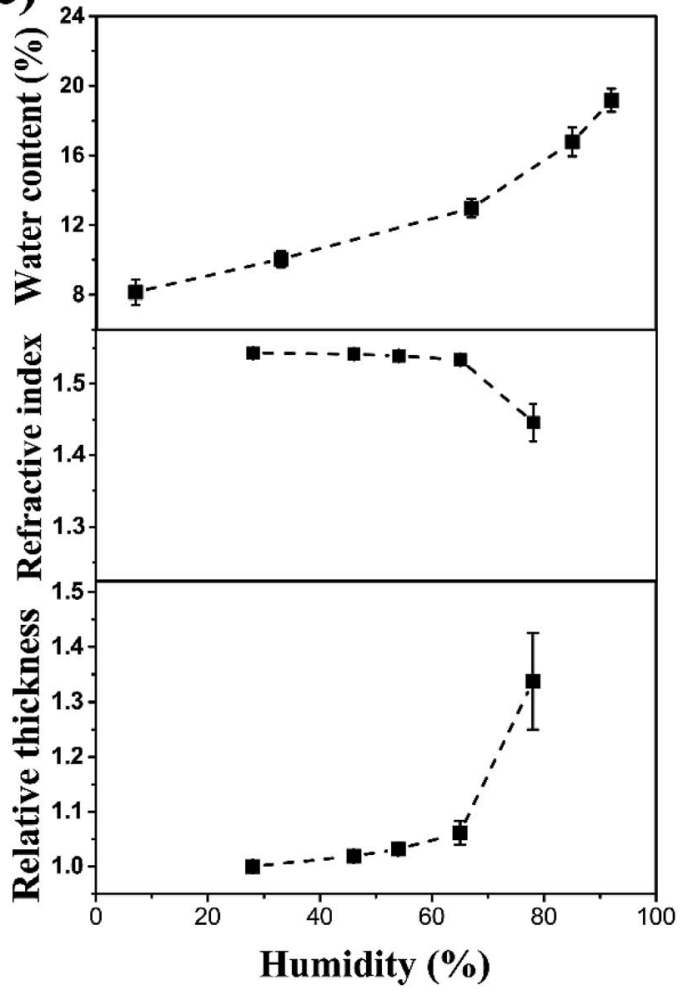

(b)

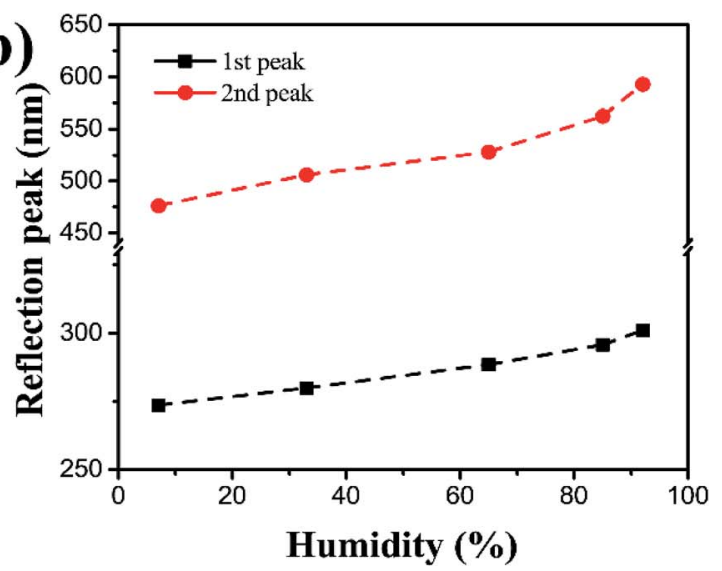

(d)

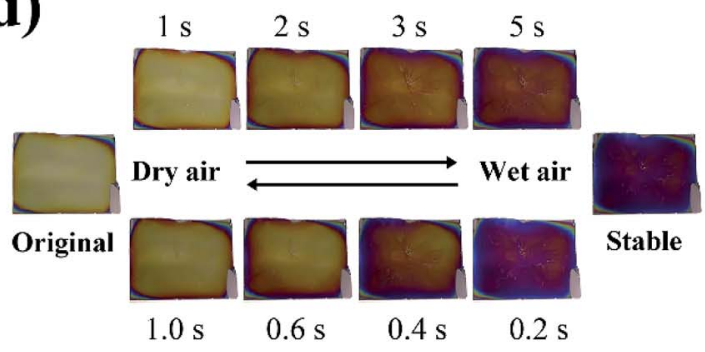

(e)

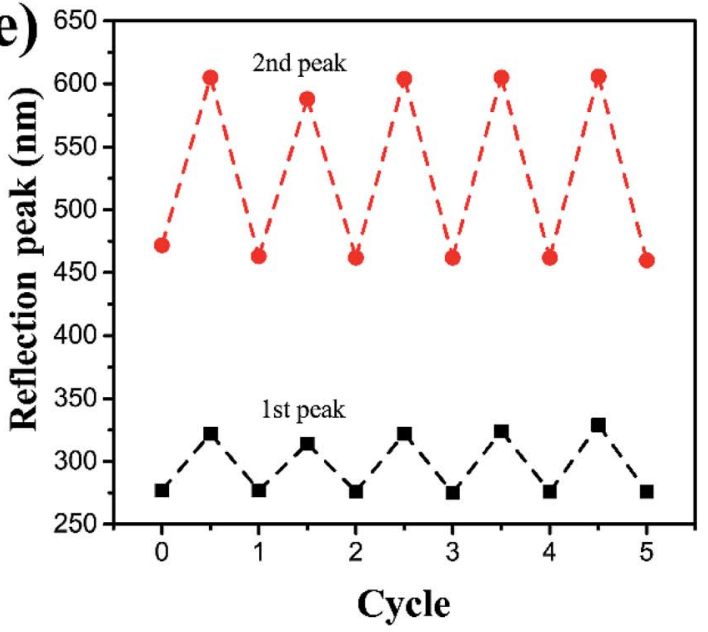

Fig. 2 Humidity-induced color change of a SF film. (a) Reflection spectra at humidity of 7\%, 33\%, 67\%, 85\%, and 92\% respectively. (b) The red shifts of two reflection peaks with increasing humidity. (c) Water uptake ability, relative thickness, and refractive index of the film at different humidity levels. (d) A cycle test of the color change between humidity levels of $\sim 10 \%$ and $\sim 90 \%$. The change can take place in $5 \mathrm{~s}$, with recovery in $1 \mathrm{~s}$ upon pumping wet or dry air. (e) Reflection peaks during 5 continuous cycles.

$92 \%$ for $2 \mathrm{~h}$. The spectrum shows two typical peaks at $273 \mathrm{~nm}$ and $476 \mathrm{~nm}$ respectively under $7 \%$ humidity. Increasing humidity resulted in clear red shifts for the two reflection peaks, accompanied with slight decreases in reflection intensity. The wavelength variations were plotted in Fig. $2 \mathrm{~b}$ as a function of humidity.

At a relatively low humidity of $<70 \%$, the peaks move to $288 \mathrm{~nm}$ and $524 \mathrm{~nm}$, and the shifts $(\Delta \lambda)$ were no larger than $20 \mathrm{~nm}$ and $50 \mathrm{~nm}$ for the first and second peaks, respectively. When the humidity was higher than $80 \%$, the peaks shifted to 50 (at $327 \mathrm{~nm}$ ) and $130 \mathrm{~nm}$ (at $624 \mathrm{~nm}$ ), representing clear color variation, which were even stronger than red shifting in some photonic crystals (the red shift is approximately $90 \mathrm{~nm}$ in ref. 36 and less than $50 \mathrm{~nm}$ in ref. 37).

The SF film physically absorbed on the Si wafer tightly, and it could not be peeled off from the substrate after drying and swelling for many times. Therefore, we believe that the size change of film in wet state mainly happened in thickness direction, while the expansion in plane direction was the minimal and negligible. The color change stems from two sources: film thickness and refractive index. When humidity increased, more water was absorbed into the SF film, consequently increasing film thickness. Below 70\% humidity, the increase in thickness was small $(\sim 5 \%$ as compared to the 
original thickness), because water uptake was limited (less than 13 wt\%), as shown in Fig. 2c. When the humidity was higher than $80 \%$, the thickness increased up to $30-35 \%$, with a water uptake of nearly $20 \mathrm{wt} \%$. The equation for Fabry-Perot interference of a dielectric thin film is the following:

$$
2 n d \cos \theta=m \lambda
$$

where $d$ is the film thickness, $n$ is the refractive index of the film, $\theta$ is the angle of incident light, $m$ is an integer, and $\lambda$ is the wavelength of light. In thin-film interference, the thickness $d$ is proportional to a half-multiple of the interference wavelength $\lambda$. Therefore, the water uptake provides a major contribution to reflection peak red shifting.

In contrast, under high humidity and with water absorbed into the film, the refractive index measured at $632 \mathrm{~nm}$ decreased from 1.54 (for pure SF) to $\sim 1.44$ (see Fig. 2c; note the refractive index for water is 1.33). According to thin-film interference, the decrease in refractive index $n$ should theoretically cause a blue shift. However, since the magnitude of refractive index decrease was much smaller (approximately 6\%) than the magnitude of thickness increase (30-35\%), the overall response showed a remarkable red shift.

Furthermore, the humidity-responsive color change of SF film is very fast, in the range of several seconds. The humidity can be rapidly changed by pumping wet air or dry nitrogen gas into the Petri dish. Wet air caused the humidity to grow very quickly, increasing to nearly $\sim 90 \%$ in tens of seconds. The corresponding color change even took place in the first $5 \mathrm{~s}$, as shown in Fig. $2 \mathrm{~d}$. When dry nitrogen was pumped to induce dryness, the film changed back to its original color even faster, at just $1 \mathrm{~s}$. The difference in response time was due to the water-contentdependent thickness change, as shown in Fig. 2c. Only upon a certain water content (about 12-15\%), the thickness change becomes much larger. Therefore, upon pumping the wet air, it takes time to reach such critical water content, while the drying makes it very rapid to decrease the water content to be below such content. Such rapid response of several seconds is much faster than many other humidity sensors, where typical response time is dozens of minutes or even longer. As all the films are only hundreds of nanometres in thickness, no difference in response time was observed for films with different thicknesses. Table 1 provides a comparison between SF films with many other multilayer or photonic humidity sensors. Considering the preparation process, this thin film has many advantages for applications in low-cost and fast humidity detections.

In addition to humidity sensitivity, the SF film also exhibited high reversibility and durability. To avoid the rapid variation of humidity and air pressure around the film, a larger chamber (a $20 \mathrm{~cm} \times 20 \mathrm{~cm} \times 20 \mathrm{~cm}$ glass box) was used to evaluate reversibility. The humidity inside the chamber was tuned from $\sim 10 \%$ to $\sim 90 \%$ by slowly pumping wet air or dry nitrogen, and the films were kept for a sufficiently long period of 1 min under each humidity condition, to allow equilibrium before each measurement. Fig. 2e presents the result of 5 cycles, showing that the reflectance peaks reversely changed with humidity. Thus, the films were concluded to have considerable reversibility and durability, and it is enough for some low-cost disposable humidity sensors.

In order to reveal the mechanism for humidity sensitivity at the molecular level, a sample of SF film was treated with ethanol to modify its crystalline structure. After being soaked in ethanol/water $(75: 25 \mathrm{v} / \mathrm{v})$, there were measurable changes in the SF film's Fourier transform infrared spectroscopy (FTIR) spectrum (Fig. 3a). The untreated film showed characteristic absorption bands at 1636,1528 , and $1235 \mathrm{~cm}^{-1}$ for the amide I, II, and III groups, representing the typical random coil protein conformation of the silk I structure. ${ }^{44}$ After the ethanol treatment, these bands shifted to 1619,1513 , and $1231 \mathrm{~cm}^{-1}$ respectively, indicating that the SF conformation had converted to the $\beta$-sheet formation of the silk II structure. ${ }^{45}$ In addition to the differences in FTIR, the X-ray diffraction (XRD) pattern showed a typical wide peak centred at $2 \theta=24.7^{\circ}$ for the untreated SF film (Fig. 3b), and a slightly narrowed peak

Table 1 A comparison of this humidity sensor with many other reported multilayer or photonic humidity sensors

\begin{tabular}{|c|c|c|c|c|c|}
\hline Structures & Materials & $\begin{array}{l}\text { Preparation } \\
\text { process }\end{array}$ & $\begin{array}{l}\text { Total response } \\
\text { time }\end{array}$ & Peak-shifts & Ref. \\
\hline Alternated thin films & PHEMA-co-PGMA, $\mathrm{TiO}_{2}$ & Layer by layer spin coating & $\sim 150 \mathrm{~s}$ & $150 \mathrm{~nm}$ & 38 \\
\hline Multilayered Bragg stacks & $\mathrm{TiO}_{2}, \mathrm{SiO}_{2}$ & Layer by layer spin coating & - & $11 \mathrm{~nm}$ & 39 \\
\hline Photonic crystal microdot & PNIPAm, poly(St-MMA-AA) particles & Inkjet printing, polymerization & $1.8 \mathrm{~s}$ & $\sim 100 \mathrm{~nm}$ & 36 \\
\hline Photonic crystal film & $\mathrm{Fe}_{3} \mathrm{O}_{4} @ \mathrm{SiO}_{2}, \mathrm{PEG}$ & $\begin{array}{l}\text { Magnetic assembly of colloidal } \\
\text { nanoparticles, photo-polymerization }\end{array}$ & $3-52 \mathrm{~min}$ & $160 \mathrm{~nm}$ & 40 \\
\hline 3D photonic crystal & $\begin{array}{l}\mathrm{Fe}_{3} \mathrm{O}_{4} @ \mathrm{C} \text { nanoparticles, } \\
\text { polyacrylamide glycol gel }\end{array}$ & $\begin{array}{l}\text { Magnetically induced self-assembly } \\
\text { of colloidal nanoparticles, radical } \\
\text { polymerization }\end{array}$ & $120 \mathrm{~min}$ & $153 \mathrm{~nm}$ & 41 \\
\hline $\begin{array}{l}\text { 3D-ordered } \\
\text { microporous film }\end{array}$ & $\mathrm{SiO}_{2}$ nanoparticles, poly(ionic liquid) & $\begin{array}{l}\text { Fabrication of opaline template, } \\
\text { infiltration of ionic liquid monomer, } \\
\text { photopolymerization, selective } \\
\text { dissolution of template }\end{array}$ & $8 \mathrm{~s}$ & $148 \mathrm{~nm}$ & 42 \\
\hline Inverse opal hydrogel & PS nanoparticles, polyacrylamide & $\begin{array}{l}\text { Fabrication of opaline template, } \\
\text { infiltration of monomer, } \\
\text { photopolymerization }\end{array}$ & $\sim 30 \mathrm{~s}$ & $35 \mathrm{~nm}$ & 43 \\
\hline One-layer thin film & Silk fibroin & Spin coating for only once & $5 \mathrm{~s}$ & $130 \mathrm{~nm}$ & $\begin{array}{l}\text { Our } \\
\text { work }\end{array}$ \\
\hline
\end{tabular}


centered at $20.7^{\circ}$ for the ethanol-treated film. It was clear that the amorphous silk I structure had converted to the $\beta$-sheet silk II structure as a result of hydrogen bond rearrangement activated by low dielectric organic solvents. ${ }^{46-48}$ Typically, the silk II crystalline region contains densely arranged $\beta$-sheet macromolecular chains, which prevents water infiltration and thus hinders film expansion. Therefore, when the humidity increased, the thickness increase for the ethanol-treated film was less than $22 \%$ (Fig. 3c). The decrease in refractive index was also smaller after the ethanol treatment, decreasing by approximately $5 \%$ from 1.55 to 1.475 . As shown in Fig. $3 d$, the wavelength changes $(\Delta \lambda)$ were approximately 25 (from $270 \mathrm{~nm}$ to $292 \mathrm{~nm}$ ) and $70 \mathrm{~nm}$ (from $479 \mathrm{~nm}$ to $548 \mathrm{~nm}$ ) for the first and second peaks, respectively, at the $92 \%$ humidity. Therefore, the ethanol-treated SF films showed much smaller red shifts than the peak shifting for the untreated SF film (approximately 50 and $130 \mathrm{~nm}$ respectively).

Fig. 4 presents a schematic illustration of the mechanism of humidity responses for SF films, proposed based on the above observations. SF is a hydrophilic-hydrophobic-hydrophilic polymer with alternating hydrophobic crystallite regions and hydrophilic amorphous blocks. ${ }^{49}$ The SF film contains amino acids with polar side groups, i.e. Ser, Tyr, Glu, and Asp, which have strong affinity to water, with the ability to absorb water molecules even in dry environments. ${ }^{50}$ In an untreated film, the fibroin polymers become entangled and form an amorphous structure; only few $\beta$-sheet crystalline domains are embedded randomly. ${ }^{51}$ According to our observations, the water absorption and film expansion are divided into two stages. In the first stage, when the humidity is lower than $70 \%$, water molecules are physically, preferentially absorbed into the pores inside of the film without breakage of chemical bonds. This results in a small volume expansion. This behaviour is similar to the reaction of the ethanol-treated film, and thus, the overall red shifting is small (Fig. 3d).

When the humidity is higher than $80 \%$, a second stage is activated, with many water molecules infiltrating into the internal pores of the cross-linked polymers. The water molecules de-bond the original hydrogen bonds between the fibroin polymers, and new hydrogen bonds form between the water molecule and the SF. ${ }^{52,53}$ The opening of cross-linked polymer chains results in volumetric expansion, therefore increasing the film thickness. However, due to the entanglement of long fibroin chains, the film cannot expand without limitation, but is restricted to a certain extent after water absorption equilibrium. Conversely, when the environment becomes drier, the infiltrated water molecules evaporate and the polymer chains crosslink again due to reformation of hydrogen bonds between the SF molecules. This process results in volume recovery and a decrease of thickness.

Ethanol treatment can modify the wetting process by increasing $\beta$-sheet crystalline domains. Following ethanol treatment, the hydrophobic blocks in the random coils of fibroin can assemble and organize to form micelles, eventually (a)

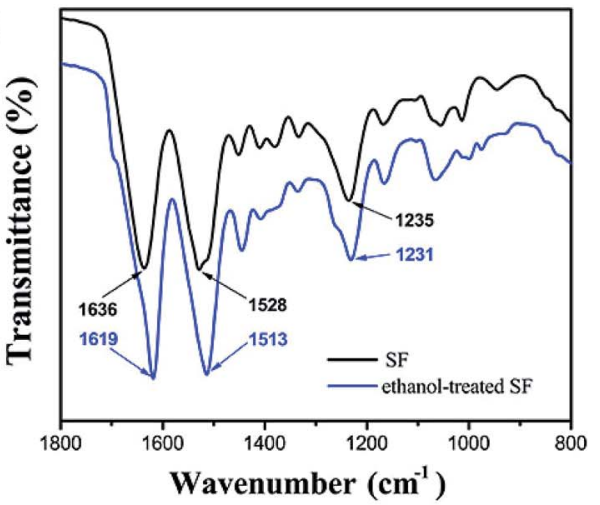

(c)

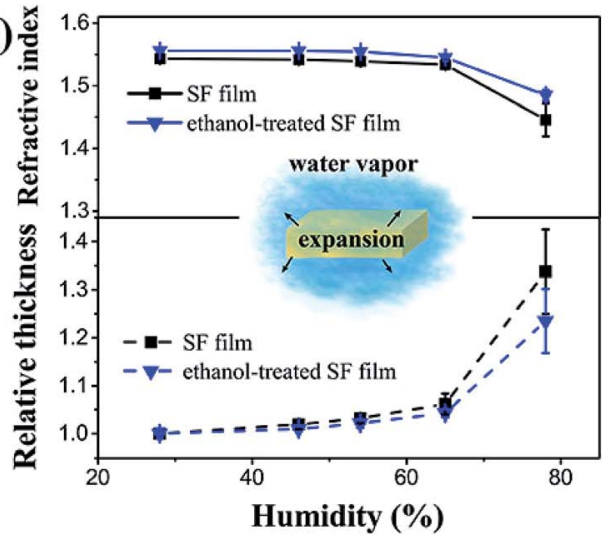

(b)

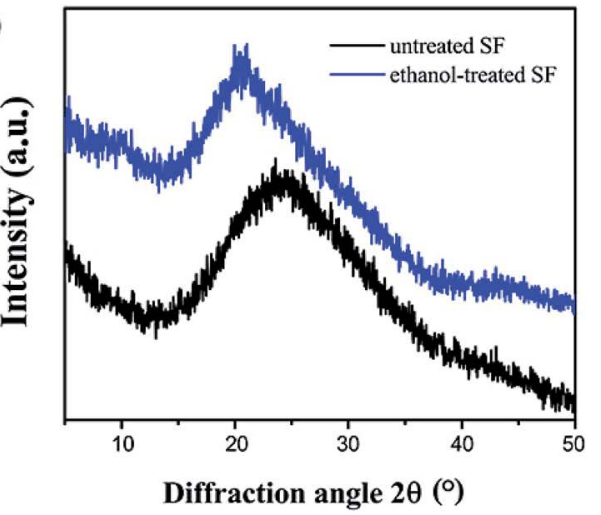

(d)

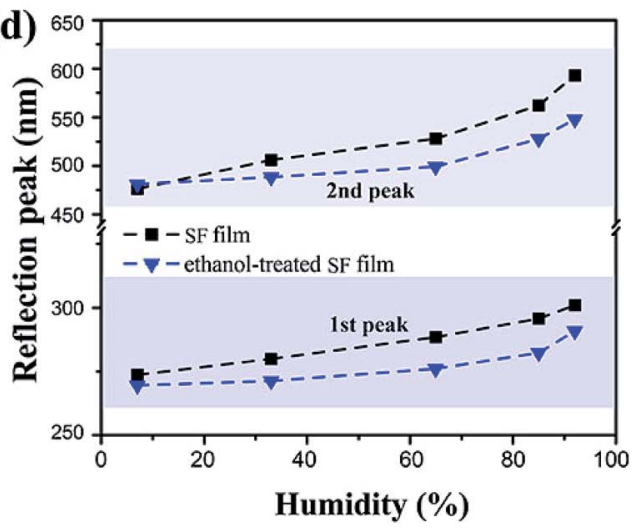

Fig. 3 Effect of ethanol treatment on the structure and optical property of the SF film: FTIR spectra (a), XRD patterns (b), refractive index and relative film thickness (c), and red shift of the two reflection peaks (d). 

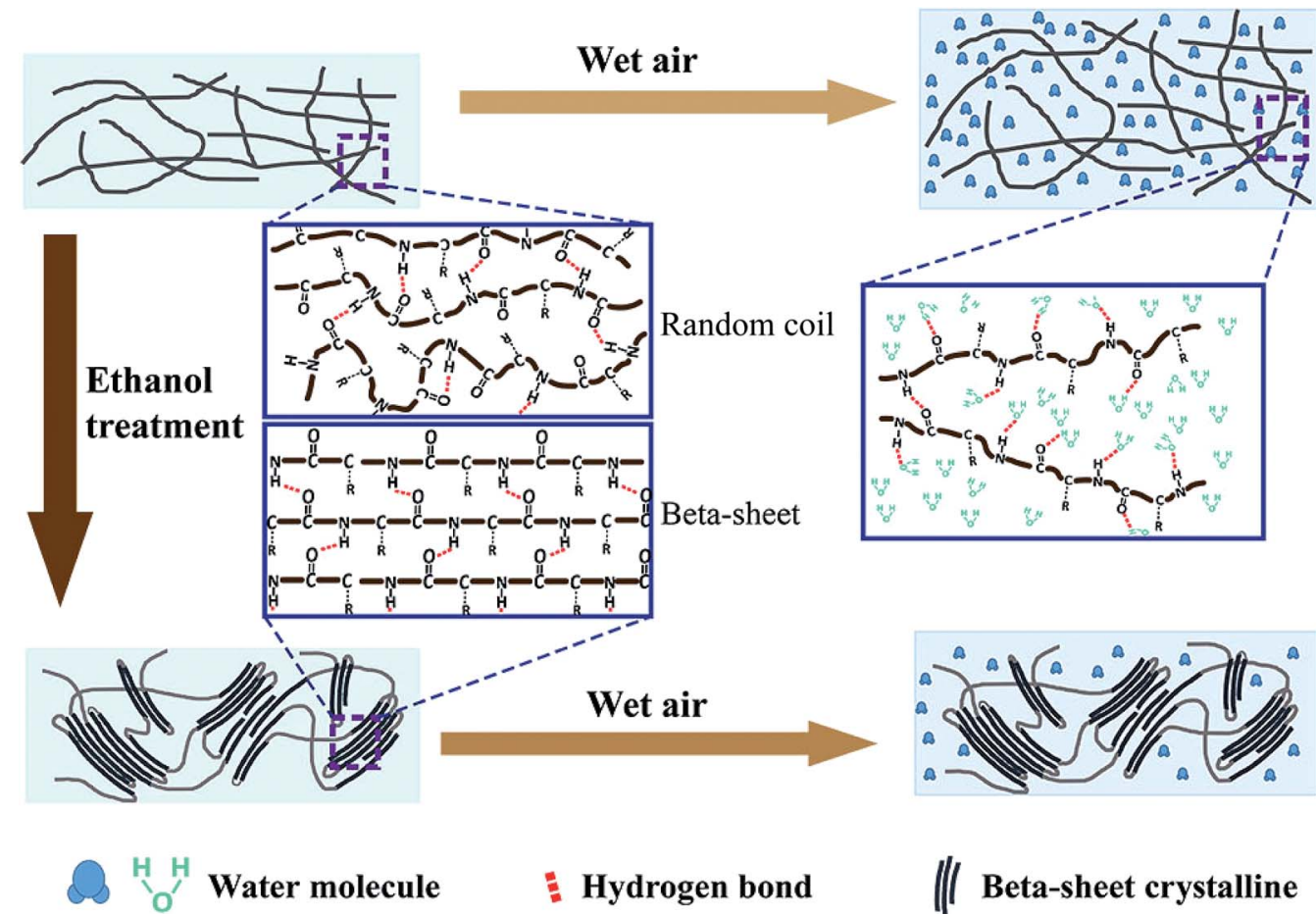

\section{Hydrogen bond}

\section{|| Beta-sheet crystalline}

Fig. 4 A schematic illustration showing the mechanism of humidity responses for the untreated and ethanol-treated SF films.

rearranging into regular and stable $\beta$-sheet crystallites. ${ }^{53}$ These insoluble crystallites can prevent the infiltration of water, subsequently hindering volumetric expansion.

Due to their humidity responsivity and color variation characteristics, the simple SF film has a promising potential in a wide range of applications. Anti-counterfeit labelling applicability was demonstrated by locally suppressing the film expansion under high humidity. Part of the film surface was masked with a hydrophobic thin film to block water infiltration; under a wet environment, the masked region maintained its original color while color changes occurred in the unmasked part. Here, the masked region was prepared by ion sputtering a thin Au film using a patterned mask (Fig. 5a). As the Au film was only $2-3 \mathrm{~nm}$ in thickness, it was almost transparent and invisible on the silk film. When the film was placed under water vapor, the thin Au film prevented the infiltration of water
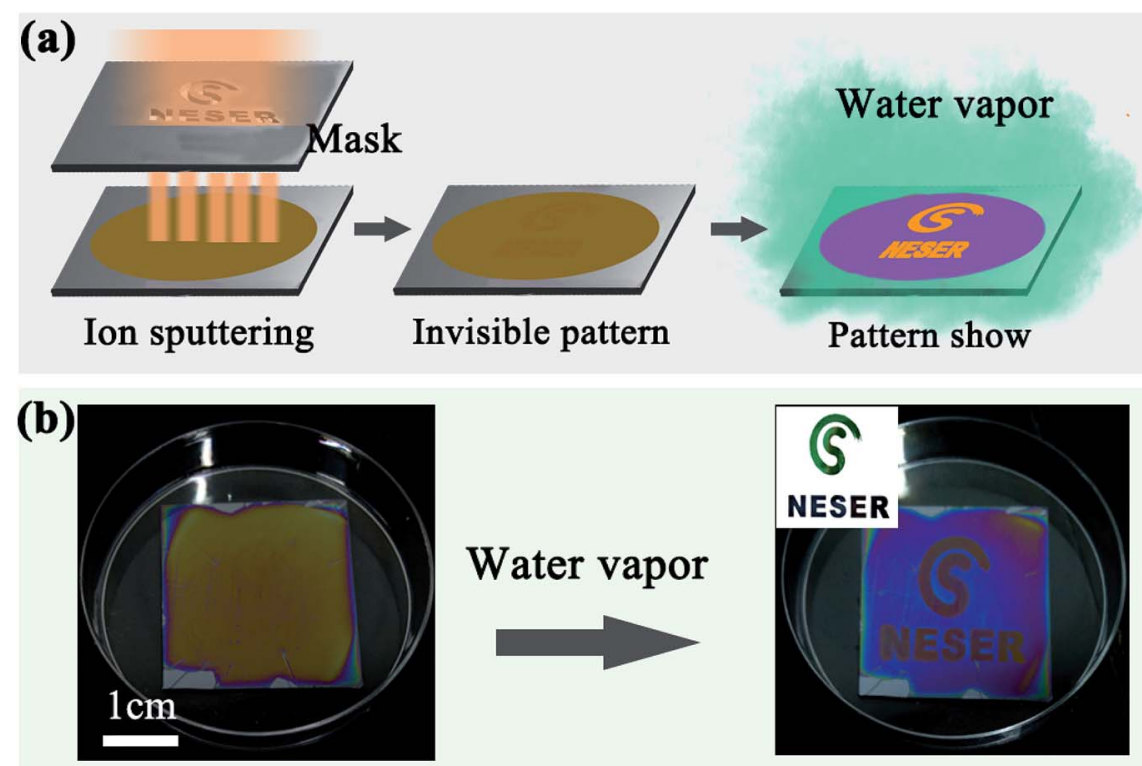

Fig. 5 Demonstration of anti-counterfeit labelling using humidity-responsive SF films. (a) Schematic illustration showing application of the anticounterfeit label on a SF film by ion sputtering. (b) The label on the SF film appears after exposure to water vapor. 
molecules, while the unmasked region expanded and quickly changed color (Fig. 5b).

\section{Conclusions}

In conclusion, we demonstrated a facile procedure to fabricate sub-micron SF films with high humidity sensitivity indicated by quick color changing. The film color was tuned by adjusting the spin coating rate, and changed rapidly in just a few seconds in response to changes in environment humidity. At higher humidity above $80 \%$, the red shift of the reflectance peak in the visible spectrum was even larger than $130 \mathrm{~nm}$, corresponding to a significant color change as distinct as yellow to violet. As the volumetric expansion was induced by the de-bonding of hydrogen bonds between fibroin polymers during the water infiltration, subsequent water evaporation did not change the intrinsic molecular structure of the polymers. Consequently, the SF film exhibited superior reversibility and durability, and the response time and peak red-shifts are superior to many other conventional structural color based humidity sensors. An anti-counterfeit labelling application was demonstrated during experimentation, showing these coloration sensors to be highly applicable in various high performance optical devices.

\section{Acknowledgements}

We gratefully thank the financial support from the National Science Foundation of China (51373110, 11404064, 51561145008, 51503137), Shanghai Pujiang Program (14PJ1401100), the Program for Professor of Special Appointment (Eastern Scholar) at Shanghai Institutions of Higher Learning, the Priority Academic Program Development (PAPD) of Jiangsu Higher Education Institutions, Qing Lan Project for Excellent Scientific and Technological Innovation Team of Jiangsu Province (2012), Project for Jiangsu Scientific and Technological Innovation Team (2013), and the Youth Innovation Promotion Association of the Chinese Academy of Sciences (2015256).

\section{Notes and references}

1 P. Ball, Sci. Am., 2012, 306, 74-79.

2 P. Vukusic and J. R. Sambles, Nature, 2003, 424, 852-855.

3 P. Vukusic, Phys. World, 2004, 17, 35-39.

4 Y. Zhao, Z. Xie, H. Gu, C. Zhu and Z. Gu, Chem. Soc. Rev., 2012, 41, 3297-3317.

5 H. Yin, B. Dong, X. Liu, T. Zhan, L. Shi, J. Zi and E. Yablonovitch, Proc. Natl. Acad. Sci. U. S. A., 2012, 109, 10798-10801.

6 Q. S. Li, Q. Zeng, L. Shi, X. H. Zhang and K. Q. Zhang, J. Mater. Chem. C, 2016, 4, 1752-1763.

7 S. Kinoshita and S. Yoshioka, ChemPhysChem, 2005, 6, 14421459.

8 A. R. Parker, R. C. McPhedran, D. R. McKenzie, L. C. Botten and N. Nicorovici, Nature, 2001, 409, 36-37.

9 D. Gur, B. Leshem, M. Pierantoni, V. Farstey, D. Oron, S. Weiner and L. Addadi, J. Am. Chem. Soc., 2015, 137, 8408-8411.
10 F. Liu, B. Q. Dong, X. H. Liu, Y. M. Zheng and J. Zi, Opt. Express, 2009, 17, 16183-16191.

$11 \mathrm{~J}$. Teyssier, S. V. Saenko, D. van der Marel and M. C. Milinkovitch, Nat. Commun., 2015, 6, 6368.

12 H. M. Princen and S. G. Mason, J. Colloid Sci., 1965, 20, 453463.

13 J. J. Richardson, M. Bjornmalm and F. Caruso, Science, 2015, 348, aaa2491.

14 L. D. Bonifacio, B. V. Lotsch, D. P. Puzzo, F. Scotognella and G. A. Ozin, Adv. Mater., 2009, 21, 1641-1646.

15 M. Kolle, A. Lethbridge, M. Kreysing, J. J. Baumberg, J. Aizenberg and P. Vukusic, Adv. Mater., 2013, 25, 22392245.

16 B. V. Lotsch, C. B. Knobbe and G. A. Ozin, Small, 2009, 5, 1498-1503.

17 J. J. Walish, Y. Kang, R. A. Mickiewicz and E. L. Thomas, Adv. Mater., 2009, 21, 3078-3081.

18 A. T. Exner, I. Pavlichenko, B. V. Lotsch, G. Scarpa and P. Lugli, ACS Appl. Mater. Interfaces, 2013, 5, 1575-1582.

19 L. Tong, W. Qi, M. Wang, R. Huang, R. Su and Z. He, Small, 2016, 12, 3433-3443.

20 Z. H. Wang, J. H. Zhang, J. X. Li, J. Xie, Y. F. Li, S. Liang, Z. C. Tian, C. A. Li, Z. Y. Wang, T. Q. Wang, H. Zhang and B. Yang, J. Mater. Chem., 2011, 21, 1264-1270.

21 M. E. Calvo, S. Colodrero, N. Hidalgo, G. Lozano, C. LopezLopez, O. Sanchez-Sobrado and H. Miguez, Energy Environ. Sci., 2011, 4, 4800-4812.

22 M. S. Park, Y. Lee and J. K. Kim, Chem. Mater., 2005, 17, 3944-3950.

23 J. A. Hiller, J. D. Mendelsohn and M. F. Rubner, Nat. Mater., 2002, 1, 59-63.

24 H. K. Raut, V. A. Ganesh, A. S. Nair and S. Ramakrishna, Energy Environ. Sci., 2011, 4, 3779-3804.

25 C. Yao, J. Ren, C. Liu, T. Yin, Y. Zhu and L. Ge, ACS Appl. Mater. Interfaces, 2014, 6, 16727-16733.

26 S. T. Parker, P. Domachuk, J. Amsden, J. Bressner, J. A. Lewis, D. L. Kaplan and F. G. Omenetto, Adv. Mater., 2009, 21, 24112415.

27 S. Ling, C. Li, K. Jin, D. L. Kaplan and M. J. Buehler, Adv. Mater., 2016, 28, 7783-7790.

28 F. G. Omenetto and D. L. KapLan, Nat. Photonics, 2008, 2, 641-643.

29 S. Kim, A. N. Mitropoulos, J. D. Spitzberg, H. Tao, D. L. Kaplan and F. G. Omenetto, Nat. Photonics, 2012, 6, 818-823.

30 H. Tao, J. M. Kainerstorfer, S. M. Siebert, E. M. Pritchard, A. Sassaroli, B. J. Panilaitis, M. A. Brenckle, J. J. Amsden, J. Levitt, S. Fantini, D. L. Kaplan and F. G. Omenetto, Proc. Natl. Acad. Sci. U. S. A., 2012, 109, 19584-19589.

31 M. Lee, H. Jeon and S. Kim, Nano Lett., 2015, 15, 3358-3363. 32 H. Kwon and S. Kim, ACS Photonics, 2015, 2, 1675-1680.

33 Y. Y. Diao, X. Y. Liu, G. W. Toh, L. Shi and J. Zi, Adv. Funct. Mater., 2013, 23, 5373-5380.

34 M. Morozova, P. Kluson, J. Krysa, P. Dzik, M. Vesely and O. Solcova, Sens. Actuators, B, 2011, 160, 371-378.

35 A. V. Yakovlev, V. A. Milichko, V. V. Vinogradov and A. V. Vinogradov, ACS Nano, 2016, 10, 3078-3086. 
36 L. B. Wang, J. X. Wang, Y. Huang, M. J. Liu, M. X. Kuang, Y. F. Li, L. Jiang and Y. L. Song, J. Mater. Chem., 2012, 22, 21405-21411.

37 L. Bai, Z. Xie, W. Wang, C. Yuan, Y. Zhao, Z. Mu, Q. Zhong and Z. Gu, ACS Nano, 2014, 8, 11094-11100.

38 Z. H. Wang, J. H. Zhang, J. Xie, C. A. Li, Y. F. Li, S. Liang, Z. C. Tian, T. Q. Wang, H. Zhang, H. B. Li, W. Q. Xu and B. Yang, Adv. Funct. Mater., 2010, 20, 3784-3790.

39 I. Pavlichenko, A. T. Exner, M. Guehl, P. Lugli, G. Scarpa and B. V. Lotsch, J. Phys. Chem. C, 2012, 116, 298-305.

40 R. Y. Xuan, Q. S. Wu, Y. D. Yin and J. P. Ge, J. Mater. Chem., 2011, 21, 3672-3676.

41 H. Hu, Q.-W. Chen, K. Cheng and J. Tang, J. Mater. Chem., 2012, 22, 1021-1027.

42 J. Huang, C. A. Tao, Q. An, C. X. Lin, X. S. Li, D. Xu, Y. G. Wu, X. G. Li, D. Z. Shen and G. T. Li, Chem. Commun., 2010, 46, 4103-4105.

43 R. A. Barry and P. Wiltzius, Langmuir, 2006, 22, 1369-1374. 44 H.-Y. Wang and Y.-Q. Zhang, Soft Matter, 2013, 9, 138-145.
45 M. Sonoyama and T. Nakano, Appl. Spectrosc., 2000, 54, 968973.

46 M. B. Dickerson, S. P. Fillery, H. Koerner, K. M. Singh, K. Martinick, L. F. Drummy, M. F. Durstock, R. A. Vaia, F. G. Omenetto, D. L. Kaplan and R. R. Naik, Biomacromolecules, 2013, 14, 3509-3514.

47 X. Chen, H. Cai, S. Ling, Z. Shao and Y. Huang, Appl. Spectrosc., 2012, 66, 696-699.

48 X. Hu, K. Shmelev, L. Sun, E.-S. Gil, S.-H. Park, P. Cebe and D. L. Kaplan, Biomacromolecules, 2011, 12, 1686-1696.

49 H.-J. Jin and D. L. Kaplan, Nature, 2003, 424, 1057-1061.

50 Z. B. Cao, X. Chen, J. R. Yao, L. Huang and Z. Z. Shao, Soft Matter, 2007, 3, 910-915.

51 Y. Cheng, L.-D. Koh, D. Li, B. Ji, M.-Y. Han and Y.-W. Zhang, J. R. Soc., Interface, 2014, 11, 20140305.

52 S. Y. Cho, Y. S. Yun, S. Lee, D. Jang, K. Y. Park, J. K. Kim, B. H. Kim, K. Kang, D. L. Kaplan and H. J. Jin, Nat. Commun., 2015, 6, 7145.

53 S. Ryu, H. H. Kim, Y. H. Park, C. C. Lin, I. C. Um and C. S. Ki, J. Mater. Chem. B, 2016, 4, 4574-4584. 